\title{
¿Cómo afecta la modalidad deportivay sus variables del entrenamiento al pulso máximo de oxígeno? How does sport and training variables affect the maximum oxygen pulse?
}

\author{
Diana Varela García, David Castiñeiras Cachaza, Jose Ángel Figueroa Domínguez \\ Universidad de ACoruña
}

\begin{abstract}
Resumen: El objeto de esta investigación ha sido el de observar el comportamiento del pulso máximo de oxígeno (que representa el consumo de $\mathrm{O}_{2}$ en cada latido) en relación con la modalidad deportiva y las variables del entrenamiento de los deportistas. El comportamiento normal del Pulso de $\mathrm{O}_{2}$ (indicador de eficiencia del sistema cardiovascular) durante una prueba de esfuerzo es el de ir incrementándose hasta llegar al esfuerzo máximo. Al observar que el pulso de $\mathrm{O}_{2}$ de una persona se estanca antes de llegar a la máxima capacidad de esfuerzo, entendemos que este cuerpo no soporta los picos máximos de intensidad en una progresión normal, pues suponemos que la progresión del entrenamiento se ha realizado demasiado rápido en referencia a este aspecto, aunque en otros mejore su rendimiento, con lo cual estamos limitando la progresión. Tras analizar una muestra de 30 individuos mediante un analizador de gases durante la realización de una prueba de esfuerzo, se han encontrado variables que tienen correlación con el pulso máximo de $\mathrm{O}_{2}$, tales como: sexo, peso, altura e intensidad. Sin embargo, no se ha encontrado correlación con el resto de variables propuestas; modalidad deportiva, edad, volumen de entrenamiento, años en la competición, horas de entrenamiento a la semana, años que se ha estado entrenado y situación competitiva actual. El grupo de deportistas que tiene más probabilidades de presentar un comportamiento del pulso de $\mathrm{O}_{2}$ creciente es el de los ciclistas, mientras que los que más probabilidades tienen de presentar un comportamiento de tipo meseta son los maratonianos.
\end{abstract}

Palabras clave: pulso; máximo; oxígeno; deportistas; prueba; esfuerzo.

Abstract: The purpose of this research was to observe the behavior of the maximum oxygen pulse (representing $\mathrm{O}_{2}$ consumption in each beat) in relation to sport and training variables of athletes. The normal behavior of the $\mathrm{O}_{2}$ pulse (efficiency indicator of cardiovascular system) during a stress test is going to grow up to the maximum stress. Noting that the $\mathrm{O}_{2}$ pulse stagnates a person before reaching maximum exercise capacity, we understand that this body does not support the current peaks in a normal progression, because we assume that the progression of training has been done too fast regarding this aspect, although in other improve their performance, which are limiting the progression. After analyzing a sample of 30 individuals using a gas analyzer for performing a stress test variables were found to correlate with the maximum $\mathrm{O}_{2}$ pulse, such as sex, weight, height and intensity. However, no correlation was found with the other proposed variables; sport, age, training volume, years in the competition, training hours per week, years that has been trained and current competitive situation.

Key words: pulse; maximun; oxygen; test; effort.

\section{Introducción}

El objeto de esta investigación ha sido el de observar el comportamiento del pulso máximo de oxígeno en relación con la modalidad deportiva y las variables del entrenamiento (frecuencia, volumen, intensidad y duración). Para ello, hemos contado con un cicloergómetro y un tapiz rodante en los que se han realizado diferentes pruebas de esfuerzo; obteniendo los datos relevantes del comportamiento del pulso de $\mathrm{O}_{2}$, que fueron tomados a cada minuto en un cuestionario que además, el paciente ha tenido que rellenar con su edad, sexo, peso, altura, modalidad deportiva, etc.

Para que esto se pueda entender un poco mejor aclararemos rápidamente lo que significa el concepto de pulso de $\mathrm{O}_{2}$. Se podría definir mediante una fórmula: $\mathrm{VO}_{2} / \mathrm{FC}$, o lo que es lo mismo, VS $x$ Dif a-v de $\mathrm{O}_{2}$. Esto quiere decir, que representa el consumo de $\mathrm{O}_{2}$ en cada latido, siendo un parámetro indicativo de la eficiencia del sistema cardiovascular. Lo que hemos buscado a lo largo de la investigación ha sido estudiar la relación entre las variables del entrenamiento (frecuencia, volumen, intensidad, duración, progresión,...) y el pulso de $\mathrm{O}_{2}$. Así que lo que creemos es que si una persona empieza a entrenar cada vez más, con una progresión muy alta sin darle tiempo a su corazón a que se adapte, llegará un momento en el que la función cardíaca no pueda seguir aumentando y el pulso de $\mathrm{O}_{2}$ se estancará.

Si durante una prueba de esfuerzo incremental, observamos como el pulso de $\mathrm{O}_{2}$ de una persona alcanza unos niveles máximos y en ese punto se estanca antes de llegar a la máxima capacidad de esfuerzo, esto nos llevará a la conclusión de que su cuerpo no es lo suficientemente capaz como para soportar esos picos de intensidad siguiendo una

Fecha recepción: 01-07-12- Fecha envío revisores: 01-07-12- Fecha de aceptación: 18-12-12 Correspondencia: Diana Varela García.

Cabría Vella (Noguerosa), S/N

CP 15609, Pontedeume, A Coruña.

Email: divarelagarcia@gmail.com progresión normal. El pulso de $\mathrm{O}_{2}$ depende del volumen sistólico y de la diferencia arterio-venosa de $\mathrm{O}_{2}$; dado que la diferencia la podemos medir con un analizador de gases, podremos centrar nuestra atención sobre el volumen sistólico, y saber de esta forma si el problema por el que el pulso no es normal viene causado por este parámetro. Esto puede ocurrir porque el corazón es un músculo y, como tal, necesita de un proceso de adaptación como cualquier otro para poder enfrentarse a nuevos estímulos que le supongan una carga mayor a la habitual. Ante esto, sería normal suponer que una progresión del entrenamiento demasiado rápida, haría mejorar algunos aspectos del rendimiento mientras que otros se «quedan atrás» y se vuelven limitantes al llegar a una carga dada. Por todo esto sería importante saber cómo se comporta y adapta el corazón ante los estímulos oportunos para saber qué es realmente lo que podemos hacer para solventar este problema.

\section{Material y método}

Los materiales utilizados para llevar a cabo la recogida de datos de esta investigación han sido los utilizados por el «Área de Medicina Deportiva» del centro de Especialidades Médicas USP Santa Teresa, entre los cuales se encuentran un analizador de gases que cuenta con una máscara especial con una turbina para medir el flujo y un cable, llamado capilar, para medir la composición del aire, un electrocardiograma de 12 derivaciones, que recogen continuamente valores sobre distintos parámetros cardiorrespiratorios $\left(\mathrm{VO}_{2}, \mathrm{VCO}_{2}, \mathrm{O}_{2} / \mathrm{HR}\right.$, RER, etc.) y un tapiz rodante o cicloergómetro, sobre los cuales se han realizado las pruebas de esfuerzo a analizar.

Para llevar a cabo este paso, diseñamos un formulario que se ha entregado a cada persona antes de realizar la prueba. En él, se nos informará sobre algunas variables que son necesarias conocer para llevar a cabo el estudio, tales como: modalidad deportiva, volumen de entrenamiento a la semana (expresado en horas y minutos), intensidades de entrenamiento (escala del 1-10 en la cual el deportista valore su 
intensidad percibida de entrenamiento), frecuencia de entrenamiento (expresado en días a la semana), además de algunos datos personales, como la edad, sexo, altura y peso. Se han recogido datos relativos a su pulso de $\mathrm{O}_{2}$ antes, durante e inmediatamente finalizada la prueba. Los datos tomados durante la prueba se han recogido al empezar cada estadío, es decir, antes del aumento del nivel de la carga externa (aumento de la velocidad en el tapiz o de la resistencia en el cicloergómetro), ya que en este momento se considera que el pulso de $\mathrm{O}_{2}$ estará más estabilizado.

Una vez que se han obtenido todos los datos de la investigación, se ha elaborado, para cada sujeto, una gráfica que representa el comportamiento del pulso de $\mathrm{O}_{2}$ a cada minuto, a lo largo de la prueba ergométrica. Cuando se han recopilado todas las gráficas, las clasificamos en 3 grupos en función de si siguen un patrón creciente, de meseta o decreciente. A continuación, se ha calculado la correlación entre esta variable y todas las demás medidas y nos centraremos en explicar aquellas correlaciones que sean más altas.

\section{Resultados y Discusión}

De las 30 gráficas analizadas hemos extraído los resultados que se muestran en la siguiente tabla.

\begin{tabular}{cccc}
\multicolumn{4}{c}{ Tabla 1. Agrupación de los deportistas según la dinámica del pulso de $\mathrm{O}_{2}$} \\
\hline PATRON & CRECIENTE & DECRECIENTE & MESETA \\
\hline $\mathrm{N}^{\circ}$ Resultados & 15 & 1 & 14 \\
\hline
\end{tabular}

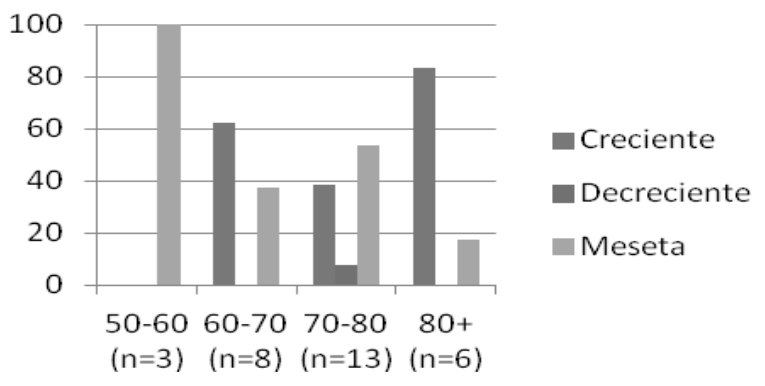

Imagen 1. Relación entre la dinámica del pulso de $\mathrm{O}_{2}$ y el peso (eje de abscisas) expresado en \% (eje de ordenadas), del total de cada subgrupo.

\section{Relación entre la dinámica del pulso de $\mathrm{O}_{2}$ y el peso}

En esta gráfica (ver imagen 1) se observa que la mayor tendencia a presentar un pulso de $\mathrm{O}_{2}$ plano la tienen aquellos sujetos de menor peso corporal, mientras que los que tienen mayor peso tienen una tendencia más alta a presentar una dinámica del tipo creciente.

Las personas con un menor pulso máximo de $\mathrm{O}_{2}$ tienen mayor tendencia a desarrollar una dinámica del pulso de $\mathrm{O}_{2}$ del tipo meseta, como veremos en el siguiente apartado, aquellos individuos con un menor peso tienen gran tendencia a tener un pulso máximo de $\mathrm{O}_{2}$ más bajo, por tanto podemos pensar, sin mucho miedo a equivocarnos, que los sujetos que tengan menor peso tendrán una mayor tendencia a desarrollar un comportamiento del pulso de $\mathrm{O}_{2}$ del tipo plano.

Relación entre la dinámica del pulso de $\mathrm{O}_{2}$ y la situación competitiva

Aquellos sujetos que compiten actualmente o que compitieron en el pasado tienen muchas más probabilidades de tener un pulso de $\mathrm{O}_{2}$ plano, mientras que casi la totalidad de los que no compiten presentan un pulso de $\mathrm{O}_{2}$ creciente. Esto nos podría sugerir que la dedicación del entrenamiento (representada por horas de entrenamiento a la semana, días de entrenamiento e intensidad) de los deportistas que compiten es la causa de que se dé esta situación, sin embargo esta hipótesis queda descartada, ya que la variables que representan la dedicación al entrenamiento han sido controladas y han mostrado una correlación baja o inexistente con esta variable (ver Imagen 2).

$\mathrm{O}_{2}$

\section{Relación entre la dinámica del pulso de $\mathrm{O}_{2}$ y el pulso máximo de}

Se puede observar (ver imagen 3) que el grupo con pulso de $\mathrm{O}_{2}$

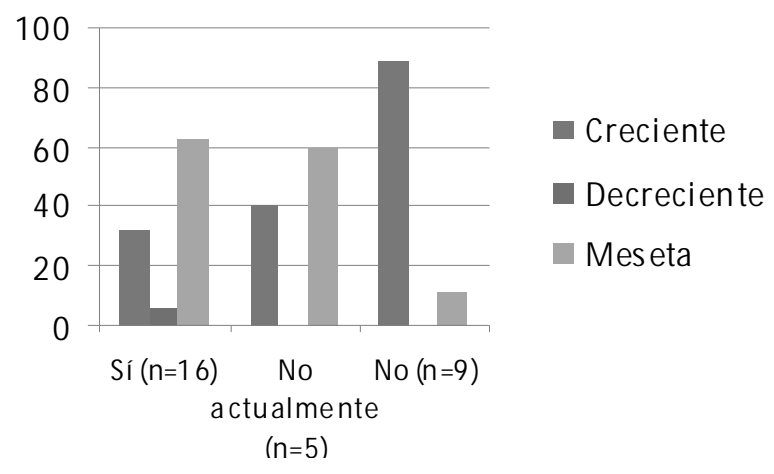

Imagen 2. Relación entre la dinámica del pulso de O2 y la situación competitiva de los sujetos (eje abscisas) expresado en \% (eje ordenadas), del total de cada subgrupo.

máximo más bajo es en el que más predomina la dinámica de tipo creciente, pero es en los grupos del medio donde se genera esta relación, casi la totalidad de la muestra del grupo 18-21 presentó una dinámica del tipo «meseta», mientras que prácticamente todos los integrantes del grupo 21-24 desarrollaron una dinámica creciente.

No se ha encontrado bibliografía específica sobre la relación entre estas variables, sin embargo, parece lógico pensar que aquellos individuos que presenten una dinámica del pulso de $\mathrm{O}_{2}$ del tipo meseta tenga un pulso máximo de $\mathrm{O}_{2}$ más bajo, ya que este pronto se estancará y dejará de aumentar.

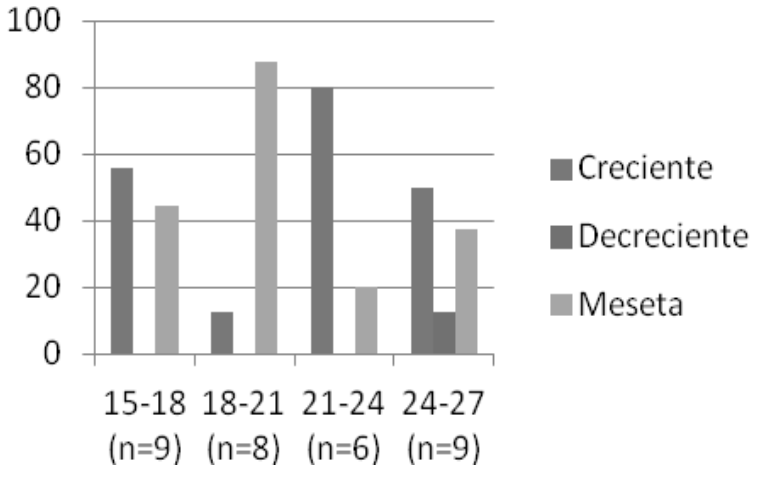

Imagen 3. Relación entre la dinámica del pulso de $\mathrm{O}_{2}$ y el pulso máximo de $\mathrm{O}_{2}$ (eje de abscisas), expresado en \% (eje de ordenadas) del total de cada subgrupo.

\section{Relación entre el comportamiento del pulso de $\mathrm{O}_{2}$ y la modalidad} deportiva

En esta gráfica (ver imagen 4) se puede observar como el grupo de deportistas que tiene más probabilidades de presentar un comportamiento del pulso de $\mathrm{O}_{2}$ creciente es el de los ciclistas, mientras que los que más probabilidades tienen de presentar un comportamiento de tipo meseta son los maratonianos. Estos resultados nos generan un

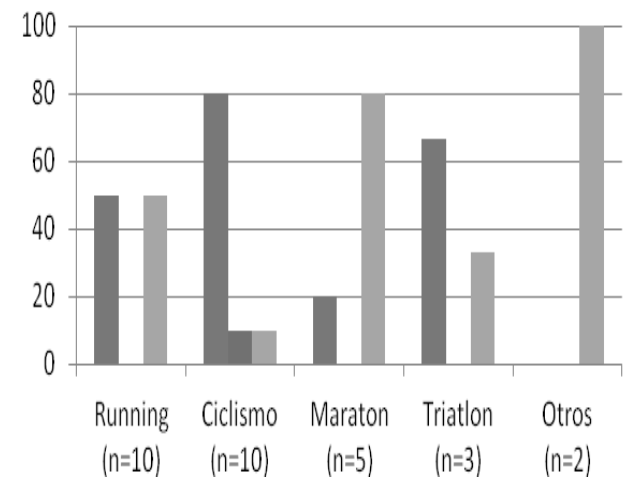

Imagen 4. Relación entre la dinámica del pulso de $\mathrm{O}_{2}$ y la modalidad deportiva practicada (eje de abscisas) expresado en \% (eje de ordenadas) del total de cada subgrupo. 
poco de confusión, ya que si viésemos los resultados obtenidos de los maratonianos sin ver los de los ciclistas pensaríamos que elevados volúmenes de entrenamiento generan una cierta tendencia a desarrollar una dinámica del pulso de $\mathrm{O}_{2}$ del tipo plano, sin embargo, si observásemos los resultados obtenidos por los ciclistas sin ver los de los maratonianos pensaríamos lo contrario, asique me he quedado un poco confusa y no sabría explicar con certeza el porquéde estos resultados.

\section{Relación entre el pulso máximo de $\mathrm{O}_{2}$ y las variables personales}

\section{Edad}

$\mathrm{Al}$ analizar esta variable con el pulso máximo de $\mathrm{O}_{2}$ se ha encontrado una baja correlación positiva, lo que nos sugiere que existe una ligera tendencia a presentar un mayor pulso máximo de $\mathrm{O}_{2}$ cuantos más años tenga el sujeto.

En la gráfica (ver imagen 5) se representa el pulso máximo de $\mathrm{O}_{2}$ medio para cada grupo de edad (intervalos de 5 años).

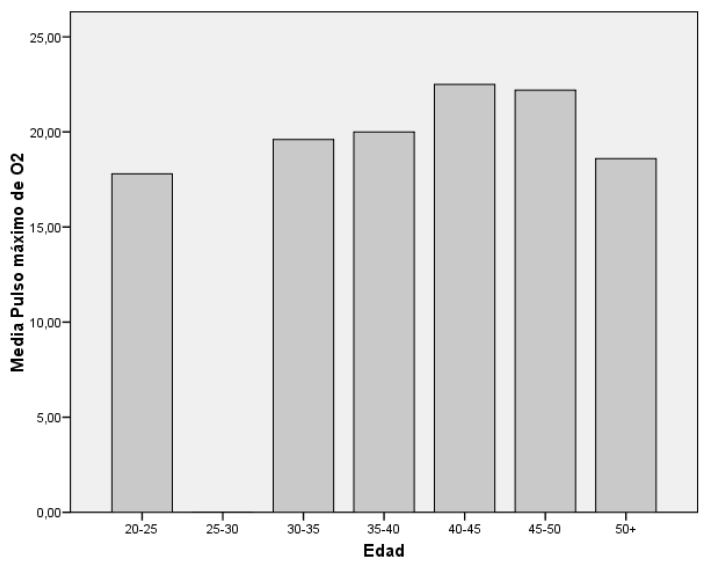

Imagen 5. Pulso máximo de $\mathrm{O} 2$ medio en los diferentes grupos de edad (intervalo 5 años).

Como se puede observar en la imagen 5 , los mayores valores del pulso máximo de $\mathrm{O}_{2}$ los alcanzan los sujetos que tienen entre 40 y 50 años, mientras que los menores valores han sido encontrados en el grupo más joven (20-25 años), seguido muy de cerca por el grupo de mayor edad (50+). Tal y como se puede ver en la imagen 6, Pimentel, Gentile, Tanaka, Seals y Gates (2003) establecen que existe una reducción del pulso de $\mathrm{O}_{2}$ con la edad, tanto en sujetos entrenados como sedentarios; aunque a cualquier edad existe un mayor pulso de $\mathrm{O}_{2}$ en los sujetos entrenados que en los sedentarios.

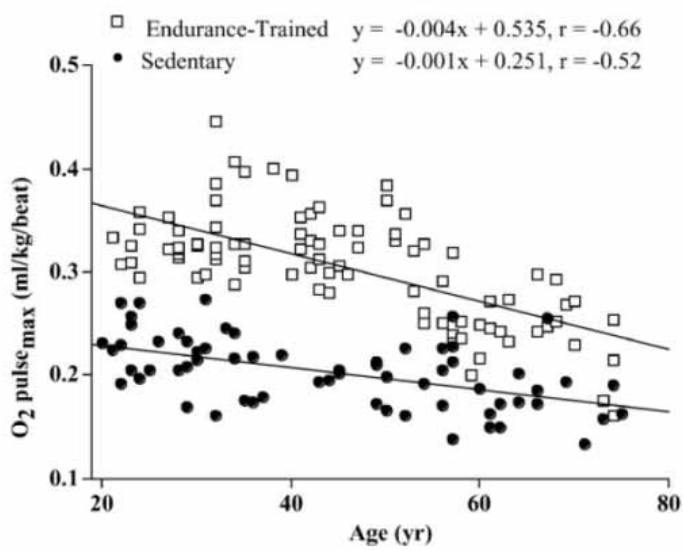

Sin embargo en este estudio la correlación entre el pulso máximo de $\mathrm{O}_{2}$ y la edad es levemente positiva, lo que nos indica que la tendencia fue que aquellos sujetos de mayor edad alcanzasen un mayor pulso de $\mathrm{O}_{2}$, lo mismo que este estudio de Pimentel et al. (2003) lo afirman Woo et al. (2006) donde hallaron que los sujetos jóvenes tenían un pulso de $\mathrm{O}_{2}$ un 14\% más elevado que los sujetos de mayor edad (20-33 años jóvenes y 25-69 los mayores). Sin embargo, no se ha encontrado bibliografía que respalde nuestra postura, lo cual sugiere que la diferencia de resultados pueda ser debida a un defecto en la muestra, como por ejemplo, el hecho de que los pocos sujetos menores de 30 años que hicieron la prueba de esfuerzo no estaban muy entrenados, quizás si se pudiera seleccionar la muestra, contando con atletas jóvenes bien entrenados, probablemente se obtuviera un resultado muy parejo a los de los estudios citados.

\section{Sexo}

Los hombres tienden a tener un mayor pulso de oxígeno máximo que las mujeres. Buena prueba de lo recién afirmado lo podemos observar tan solo con ver la media del pulso máximo de $\mathrm{O}_{2}$ de ambos grupos, este valor es de $16,1 \mathrm{ml}_{\text {.lat }}{ }^{-1}$ para las mujeres, y de $21,6 \mathrm{ml}_{\text {.lat }}{ }^{-1}$ para los hombres; con esto se ve que los hombres tienden a tener un mayor pulso máximo de $\mathrm{O}_{2}$ sin necesidad de hacer ninguna correlación. Para más certeza, en la imagen 7 se ve una gráfica representativa de este punto.

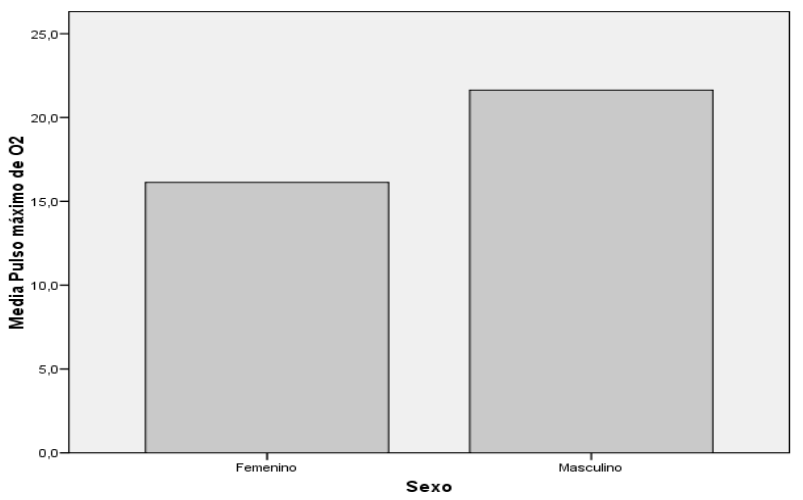

Imagen 7. Pulso máximo de $\mathrm{O}_{2}$ medio de hombre y mujeres.

En la imagen 7 se puede apreciar con total claridad las diferencias, que alcanzan un $34 \%$ a favor del grupo masculino.

Un estudio de Woo et al. (2006) analizaba, entre otras cosas, el pulso máximo de $\mathrm{O}_{2}$ alcanzado durante una prueba de esfuerzo máximo en hombres y mujeres, en este estudio tuvo como resultado que los hombres alcanzaron un pulso de $\mathrm{O}_{2}$ máximo un $49 \%$ mayor que las mujeres. Si hacemos un poco de memoria la diferencia en nuestro estudio entre hombres y mujeres fue del $34 \%$, esta diferencia entre los hallazgos encontrados en este estudio y el de Woo probablemente sean debidas a que en nuestro caso los sujetos de estudio eran deportistas activos, mientras que en estudio recién citado eran sedentarios, además, por tanto creemos que si en nuestro estudio los sujetos fuesen sedentarios quizás las diferencias entre el pulso máximo de $\mathrm{O}_{2}$ entre ambos sexos se acercarían más a las halladas en el estudio de Woo et al. (2006).

\section{Peso}

En esta relación es en donde se ha dado la correlación más alta (es positiva), por lo que en este caso se experimentará un aumento

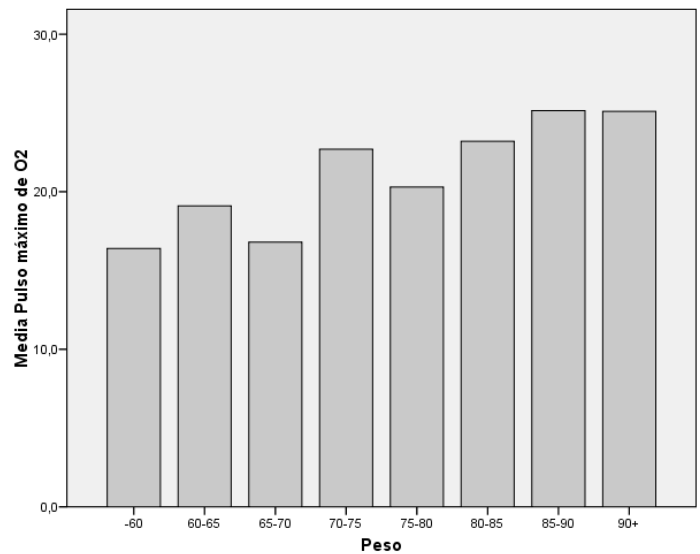

Imagen 8. Pulso máximo de $\mathrm{O}_{2}$ alcanzado por cada grupo de peso (intervalo $5 \mathrm{~kg}$.) 
considerable del pulso máximo de $\mathrm{O}_{2}$ cuanto más elevado sea el peso. En este caso se han dividido los sujetos en 8 grupos en función del peso (intervalo 5kg), al hallar el valor medio del pulso máximo de $\mathrm{O}_{2}$ de cada grupo se ha obtenido el siguiente resultado (ver imagen 8).

Como se puede observar, la tendencia sigue siendo que conforme aumenta el peso también lo hace el pulso máximo de $\mathrm{O}_{2}$, aunque existe algunabajada.

En la bibliografía consultada no se han encontrado investigaciones en las que se haya relacionado directamente el pulso máximo de $\mathrm{O}_{2}$ y el peso corporal, sin embargo, estos datos los podemos obtener de forma indirecta, ya que como sabemos el pulso máximo de $\mathrm{O}_{2}$ es igual al $\mathrm{VO}_{2}$ absoluto entre la FC. Así Batterhamn, Vanderburgh, Mahar y Jackson (1999) encuentran que hay una relación positiva entre el peso de los sujetos y el $\mathrm{VO}_{2}$ máximo absoluto (ver imagen 9).

En la imagen 9 podemos observar como el $\mathrm{VO}_{2}$ absoluto máximo tiende a ser mayor cuanto más elevado es el peso de los sujetos. Otra gráfica representativa de recientemente se ha afirmado la exponen LeMaitre, Harris, Hannan, Fox y Denvir (2005), con un resultado muy similar, aunque con la diferencia de que en este caso sólo se tiene en cuenta la masa muscular, en vez de la masa corporal total, sin embargo observamos que la tendencia es la misma.

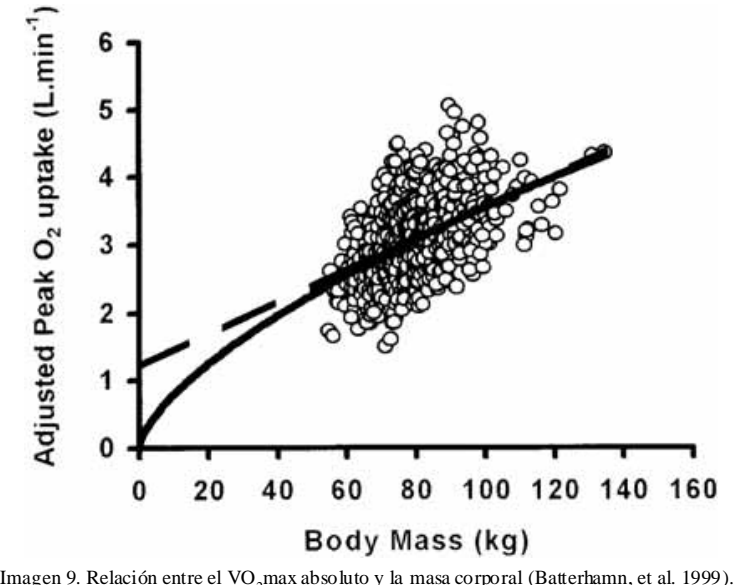

Queda claro que el $\mathrm{VO}_{2}$ máximo absoluto está muy influenciado por el peso del sujeto (ver imagen 10 ). Si el $\mathrm{VO}_{2}$ máx absoluto aumenta con el peso mientras que la FC no cambia, el resultado será un pulso máximo de $\mathrm{O}_{2}$ más elevado para los sujetos de más peso, tal y como dicen los resultados obtenidos en este estudio.

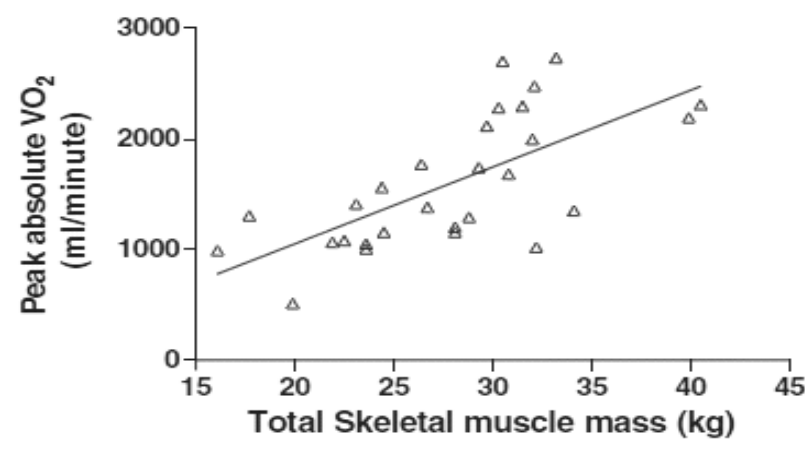

Imagen 10. Relación entre el $\mathrm{VO}_{2}$ max absoluto y la masa muscular esquelética (LeMaitre, et al., 2005).

\section{Altura}

Esta relación ha sido una de la cual se ha sacado una correlación más elevada, lo que nos dice que hay grandes probabilidades de que los sujetos más altos sean los que alcancen durante la prueba ergométrica unos valores de pulso máximo de $\mathrm{O}_{2}$, más elevados.

$\mathrm{Al}$ a agrupar a los individuos en 5 grupos en función de su altura (intervalo $5 \mathrm{~cm}$ ) pudimos obtener la siguiente gráfica representativa del

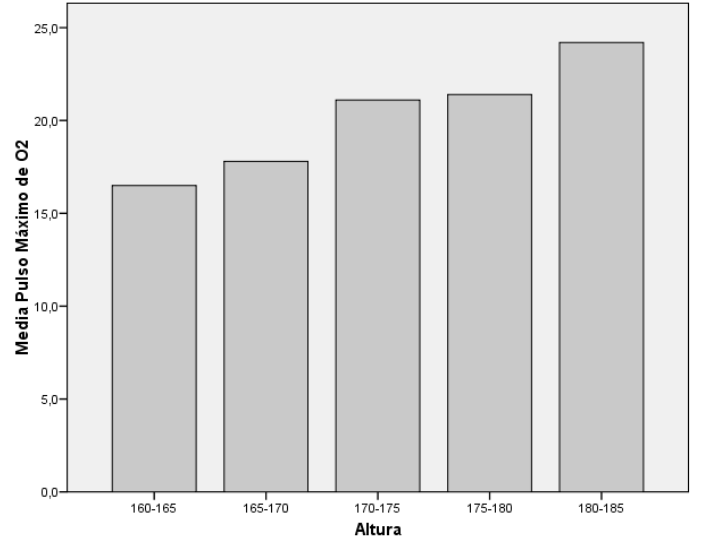

Imagen 11. Pulso máximo de $\mathrm{O}_{2}$ medio alcanzado por cada grupo de altura (intervalo $5 \mathrm{~cm}$.).

pulso máximo de $\mathrm{O}_{2}$ medio alcanzado por cada grupo (Ver imagen 11).

Al igual que en el caso del peso, en la literatura consultada no se ha encontrado nada escrito que relacione a la altura con el pulso máximo de $\mathrm{O}_{2}$, sin embargo, tras lo mencionado en el anterior apartado sobre la relación peso-pulso máximo de $\mathrm{O}_{2}$ consideramos que es suficiente con explicar la relación entre el peso y la altura, que como bien se sabe, a mayor altura más masa corporal, por tanto mayor peso, la siguiente tabla así lo demuestran (Ver tabla 2).

\begin{tabular}{|c|c|c|c|c|c|}
\hline $\begin{array}{c}\text { Altura } \\
(\mathrm{cm})\end{array}$ & $\begin{array}{c}\text { Peso mínimo } \\
(\mathrm{kg})\end{array}$ & $\begin{array}{c}\text { Peso máximo } \\
(\mathrm{kg})\end{array}$ & $\begin{array}{c}\text { Altura } \\
(\mathrm{cm})\end{array}$ & $\begin{array}{c}\text { Peso mínimo } \\
(\mathrm{kg})\end{array}$ & $\begin{array}{c}\text { Peso máximo } \\
(\mathrm{kg})\end{array}$ \\
\hline 152.4 & 45 & 69,4 & 179 & 55,8 & 89,1 \\
\hline 153,7 & 45 & 69,9 & 180 & 57.6 & 90,3 \\
\hline 154,9 & 46 & 70,3 & 182 & 57,6 & 91,9 \\
\hline 156.2 & 46 & 71.0 & 183 & 59.4 & 93,0 \\
\hline 157.5 & 47 & 71,7 & 184 & 59,4 & 94,3 \\
\hline 158.8 & 47 & 72,1 & 185 & 61.2 & 95,7 \\
\hline 160.0 & 47 & 72,6 & 187 & 61,2 & 97,3 \\
\hline 161,3 & 47 & 73,5 & 188 & 63,1 & 98,9 \\
\hline 162.6 & 48 & 74,4 & 189 & 63,1 & 100,2 \\
\hline $\begin{array}{l}162.6 \\
163.8\end{array}$ & $\begin{array}{l}48 \\
48\end{array}$ & $\begin{array}{l}75,4 \\
75,5\end{array}$ & $\begin{array}{l}189 \\
191\end{array}$ & $\begin{array}{l}64,1 \\
64,9\end{array}$ & 101,6 \\
\hline 165.1 & 48 & 76,7 & 192 & 64,9 & 103,0 \\
\hline 166,4 & 48 & 77,8 & 193 & 66,7 & 104,3 \\
\hline 167,6 & $\begin{array}{l}48 \\
49\end{array}$ & 78,9 & 194 & 66,7 & 105,7 \\
\hline 168.9 & 49 & 80,1 & 196 & $\begin{array}{l}08,1 \\
68,5\end{array}$ & 107,0 \\
\hline 170.2 & 50 & 81,2 & 197 & 68,5 & 108,4 \\
\hline 171.5 & 51 & 82,3 & 198 & 69.4 & 109,8 \\
\hline 172.7 & 52 & 83,5 & 199 & 69,4 & 111,1 \\
\hline 174,0 & 52 & 84,6 & 201 & 71,2 & 112,5 \\
\hline 175.3 & 54 & 85,7 & 202 & $\begin{array}{l}1,2 \\
71,2\end{array}$ & 113,9 \\
\hline 176,5 & 54 & 86,9 & 203 & 73,0 & 115,2 \\
\hline 177.8 & 56 & 88,0 & 204 & 73,0 & 116,6 \\
\hline
\end{tabular}

permisible; por cada $2,5 \mathrm{~cm}$ por encima de $200 \mathrm{~cm}$, añada $2,7 \mathrm{~kg}$ hasta el peso máximo

En la tabla 2 se observa como los rangos saludables de peso incrementan a medida que aumenta la altura, por tanto si retomamos y volvemos al apartado anterior, donde se argumentó que un mayor peso implica un mayor pulso máximo de $\mathrm{O}_{2}$, quedará también explicado que una mayor altura implicará un mayor pulso máximo de $\mathrm{O}_{2}$.

Relación entre el pulso máximo de $\mathrm{O}_{2}$ y las variables del entrenamiento

\section{Años en la competición}

$\mathrm{Al}$ analizar esta variable con el pulso máximo de $\mathrm{O}_{2}$ se ha obtenido una baja correlación negativa, lo cual nos dice que existe una mínima tendencia a que haya un menor pulso de $\mathrm{O}_{2}$ en aquellos sujetos que llevan muchos años en la competición. Para analizar mejor esta relación se han agrupado a los sujetos en 5 grupos en función de los años que llevan entrenando (intervalo 3 años), la siguiente gráfica muestra la media de pulso máximo de $\mathrm{O}_{2}$ en cada grupo (Ver imagen 12).

Esta relación nos indica que los cambios que suceden en el pulso máximo de $\mathrm{O}_{2}$ en función de los años que lleva el sujeto en la competición no son muy notables, por lo que esta variable no es muy importante.

No se ha encontrado bibliografía referente a esta relación.

Intensidad de entrenamiento percibida por el sujeto

Se ha encontrado una correlación mediana entre esta variable y el pulso máximo de $\mathrm{O}_{2}$, lo que nos dice claramente que en aquellos sujetos que entrenen a una intensidad mayor será más probable observar un 


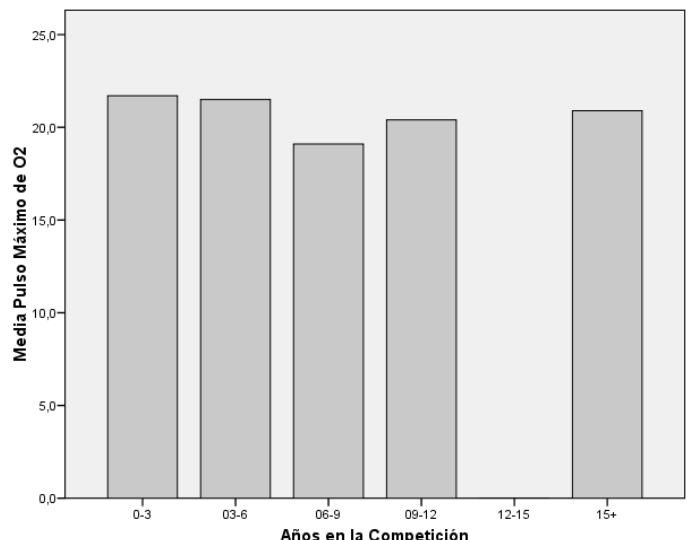

Imagen 12. Pulso máximo de $\mathrm{O}_{2}$ en cada grupo según los años que lleva compitiendo (intervalo 3 años)

pulso máximo de $\mathrm{O}_{2}$ más elevado. En este caso los sujetos fueron agrupados según la intensidad que para ellos tienen sus entrenamientos, el pulso máximo de $\mathrm{O}_{2}$ medio de cada grupo se contempla en la siguiente gráfica(Ver imagen 13).

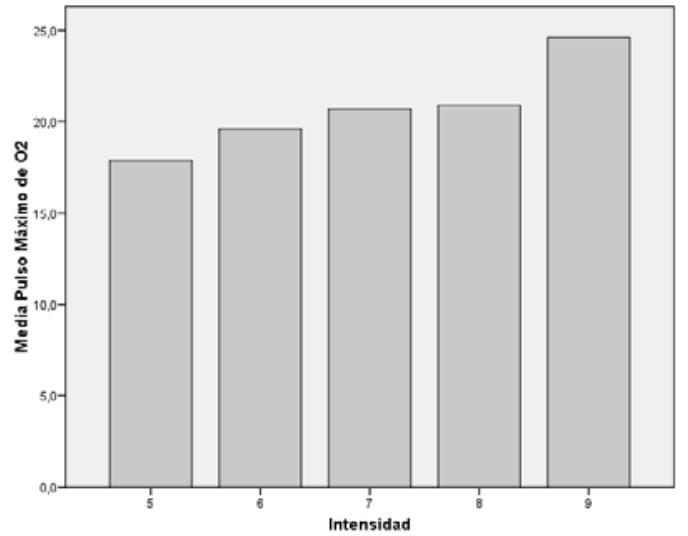

Imagen 13. Pulso máximo de $\mathrm{O}_{2}$ en cada grupo según la intensidad au topercibida de sus

No hay nada escrito específicamente sobre la influencia de la intensidad del entrenamiento sobre el pulso máximo de $\mathrm{O}_{2}$ aun así podemos deducir esta relación a través de los resultados de las diferentes modalidades deportivas, ya que sabemos que no es igual la intensidad del entrenamiento de un maratoniano, que acostumbra a correr cerca del $70 \%$ de su $\mathrm{VO}_{2}$ máx (ritmo de competición), a la de un atleta de $400 \mathrm{~m}$ lisos, cuya intensidad de competición puede alcanzar el 120\% de su $\mathrm{VO}_{2}$ máx, por tanto queda claro que son las disciplinas de más corta duración las que alcanzan una mayor intensidad de entrenamiento, sin embargo, al revisar la bibliografía, podemos encontrar que miembros del equipo griego de halterofilia tienen un pulso máximo de $\mathrm{O}_{2}$, levemente superior al de individuos sedentarios (Anastasakis, et al., 2005), 14,9ml.lat ${ }^{1}$, que un atleta de élite de $400 \mathrm{~m}$. lisos lo tiene en torno a $18 \mathrm{ml}$. lat $^{1}$ (Padilla, et al., 2000) o que un gimnasta de élite lo puede tener, también, cerca de los 28ml.lat ${ }^{-1}$ (Barbier, et al., 2006), mientras que, como ya vimos en un anterior apartado, un ciclista puede llegar a los 27,3ml.lat ${ }^{1}$ (Barbier, et al., 2006) y un atleta de fondo a 25,3ml.lat ${ }^{1}$ (Anastasakis, et al., 2005), analizados estos resultados hay algo que no concuerda con los obtenidos en nuestro estudio, y es que si estimásemos la intensidad de entrenamiento de estos y calculásemos la correlación entre intensidad y pulso máximo de $\mathrm{O}_{2}$ mediante la correlación bivariada de Pearson el resultado sería, con toda seguridad, negativo, mientras en nuestro estudio fue positivo de 47.

¿Cuál es entonces el problema? Creemos que el principal problema que nos lleva a este desajuste es una confusión de los sujetos sobre el término «intensidad» (que por supuesto es culpa nuestra por no haberlo dejado claro), y es que muchos, o la mayoría lo han confundido con la carga de entrenamiento, este factor depende de la intensidad, volumen y densidad (García-Verdugo, 2008), por tanto ya no es la intensidad lo que valoran, de ahí se puede extraer el por qué un maratoniano ha valorado su intensidad de entrenamiento con un 9 sobre 10 (lo que sería un 90\%), aún no existe el deportista que aguante esta intensidad durante tanto tiempo.

\section{Situación competitiva actual}

En este caso ha sido hallada una correlación negativa, lo que indica que aquellos sujetos que compiten tienen más probabilidades de alcanzar un pulso máximo de $\mathrm{O}_{2}$, mayor que los que no compiten. Para esta relación sólo se han hecho 3 grupos (Sí, No, No actualmente), el pulso máximo de $\mathrm{O}_{2}$ alcanzado por cada grupo se puede contemplar en la siguiente gráfica (ver imagen 14$)$.

En la imagen 14se contempla que el grupo con mayor pulso máximo

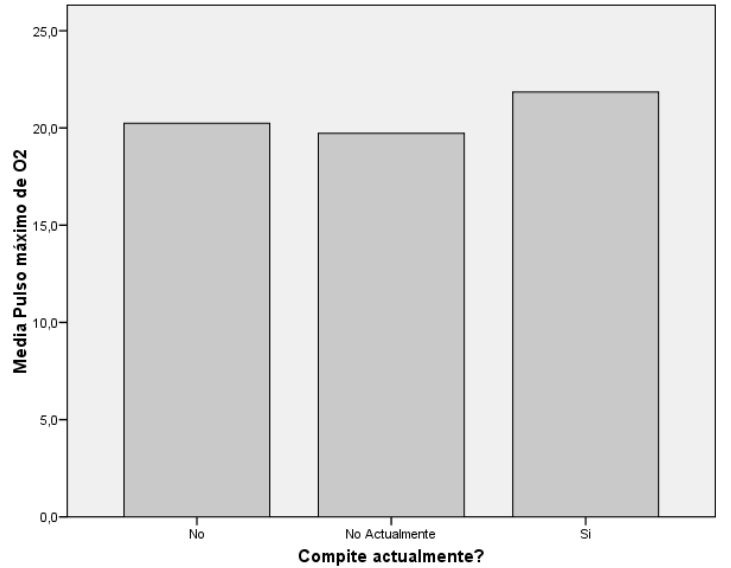

Imagen 14. Pulso máximo de $\mathrm{O}_{2}$ alcanzado en función de la situación competitiva.

de $\mathrm{O}_{2}$ es el que actualmente compite. La desviación típica es 3.70 (no), 3.94 (no actualmente) y 2.79 (Sí).

No se ha encontrado bibliografía que contemple esta relación, las comparaciones más similares que se han podido encontrar han sido entre individuos entrenados y desentrenados en publicaciones de Padilla et al. (2000), Barbier et al. (2006), Anastasakis et al. (2005), Sharma et al. (2000), etc. Evidentementeen todos ellos se apreciauna gran diferencia entre el pulso máximo de $\mathrm{O}_{2}$ de sujetos entrenados y no entrenados, a favor del primer grupo, sin embargo estos datos no servirían para contrastarlos con los obtenidos en este estudio, por tanto no merece la pena citarlos.

\section{Modalidad deportiva}

Los resultados encontrados sobre la modalidad deportiva seexpresan en la siguiente gráfica (ver imagen 15).

Como se puede observar en la imagen 15 la modalidad deportiva en la cual se encuentra un mayor pulso de $\mathrm{O}_{2}$ es el maratón (22.6ml.lat ${ }^{1}$ ), seguida muy de cerca por el ciclismo (20 ml.lat ${ }^{1}$ ), más alejado se encuentra el pulso máximo de $\mathrm{O}_{2}$ del triatlón (20.6ml.lat ${ }^{-1}$ y los valores más bajos los recogen los del grupo de running (19.6ml.lat $\left.{ }^{1}\right)$ y otros. Mientras que la desviación típica encontrada fue de 3.16 para running; 1.21 para triatlón; 3.99 para maratón; 3.77 para el ciclismo y 3.18 para «otros».

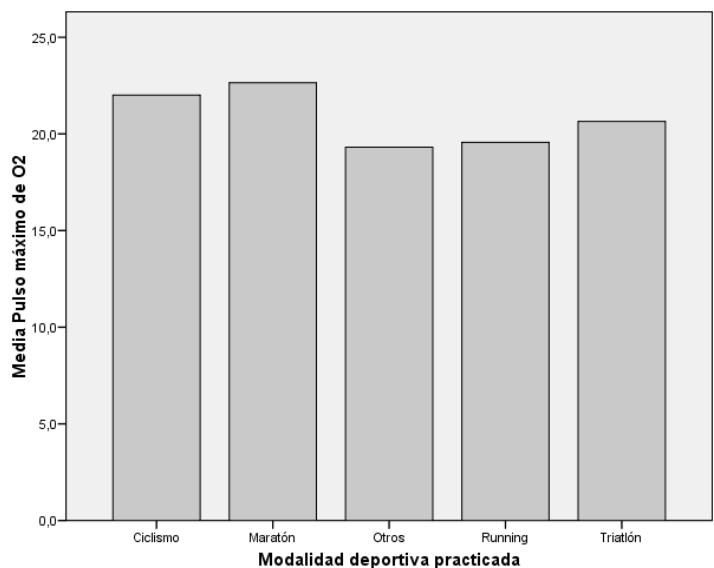

Imagen 15. Pulso máximo de $\mathrm{O}_{2}$ medio en las diferentes modalidades deportivas. 
Como ya se mostró en la tabla, la relación que existe entre el pulso máximo de $\mathrm{O}_{2}$ y esta variable es muy baja, lo que nos indica que los valores más altos de pulso de oxígeno tenderían a encontrarse en la modalidad deportiva de maratón (valor asignado 4) pero esta correlación queda muy neutralizada porque el valor asignado al ciclismo fue 1 .

Para esta variable se ha realizado la prueba de la mediana, cuyos resultados son expuestos en la siguiente tabla (Ver tabla 3).

\begin{tabular}{cccccc}
\multicolumn{7}{c}{ Tabla 3. Resultados de la prueba de la mediana. } \\
\hline \multicolumn{5}{c}{ Deporte } \\
\hline Pulso máximo de $\mathrm{O}_{2}$ & Running & Ciclismo & Triatlón & Maratón & Otros \\
\hline$>$ Mediana & 47 & 145 & 22 & 74 & 21 \\
= Mediana & 146 & 70 & 40 & 36 & 17 \\
\hline
\end{tabular}

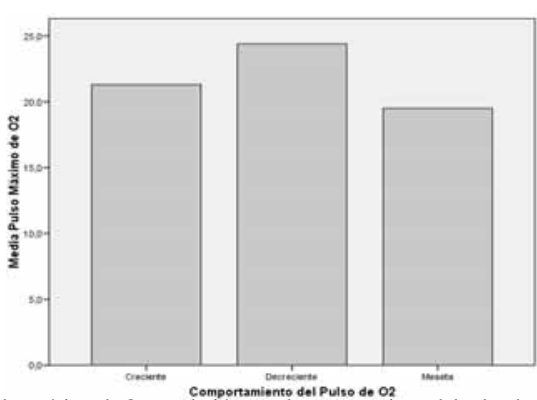

Imagen 16. Pulso máximo de $\mathrm{O}_{2}$ en relación con el comportamiento del pul so de $\mathrm{O}_{2}$ a lo largo de la prueba de esfuerzo.

Lo que nos quiere decir que para el caso del running que sólo el $24.3 \%$ de los casos se hayan por encima de la media y el $75.7 \%$ restable por debajo, en el ciclismo encontramos que el $67.4 \%$ de los casos están por encima de la mediana y el 32.6\% restante por debajo, en el triatlón encontramos sólo un $35.5 \%$ de los casos por encima de la mediana y un $64.5 \%$ por debajo, en maratón el $67.3 \%$ de los casos por encima de la mediana y el $32.7 \%$ por debajo de la misma y, por último, en el grupo designado «otros» vemos que el $55.3 \%$ de los casos están por encima de la mediana, mientras que el $44.7 \%$ restante se haya por debajo.

El estudio de Padilla et al.(2000) obtuvo los resultados más elevados de pulso máximo de $\mathrm{O}_{2}$ en los deportistas maratonianos (22ml.lat ${ }^{1}$ ), resultados muy parejos a los nuestros, donde también son los maratonianos los que alcanzan un mayor pulso máximo de ${ }_{2}$ (22.6ml.lat 1). Sin embargo, se vuelve más difícil la comparación entre el resto de los grupos de deportistas evaluados ya que sus modalidades deportivas no coinciden; aun así se puede observar que en el estudio de Padilla los resultados más bajos fueron de los grupos cuya modalidad deportiva requiere un menor tiempo de actividad (karate y 1500m lisos), es decir, al igual que en nuestro estudio, ya que encontramos los valores más reducidos en los grupos running (que desarrollan un menor volumen de entrenamiento que el resto de los grupos) y «otros» (formados por un jugador de tenis de mesa y otro de fútbol playa).

Otro estudio comparable con el nuestro es el de Barbier et al. (2006) en el que sólo incluyó a gimnastas, canoistas y ciclistas, donde el grupo de ciclistas resultó ser el que obtuvo un valor más elevado de pulso de $\mathrm{O}_{2}$ (27 ml.lat ${ }^{1}$. Tal y como podemos observar en la imagen 15, en nuestro estudio el grupo de ciclistas también se sitúa en la parte más alta, sin embargo en nuestro caso el pulso máximo de $\mathrm{O}_{2}$ tan sólo alcanza los $22 \mathrm{ml} . \mathrm{lat}^{1}$; esta diferencia del $24 \%$ entre nuestros ciclistas y los del estudio de Barbier probablemente se deba a que en su estudio los ciclistas pertenecían a la élite nacional, mientras que en el nuestro la mayoría lo hace como una forma de ocio y salud. Resultados muy similares a los Barbier et al. (2006) los encontramos también en un estudio de Anastasakis y cols. (2005), donde una vez más vemos que los atletas dedicados a modalidades deportivas de resistencia son los que alcanzan los mayores valores de pulso máximo de $\mathrm{O}_{2}\left(25.3 \mathrm{ml}\right.$. lat $^{-1}$ para atletas de larga y media distancia). Al igual que en el caso del estudio de Barbier, en el deAnastasakis también se contó con deportistas de alto nivel nacional (selección griega de atletismo) lo cual puede justificar los mayores valores del pulso máximo de $\mathrm{O}_{2}$ encontrados en sendos estudios.

Dinámica del pulso de $\mathrm{O}_{2}$ durante el ejercicio

La correlación entre esta variable y el pulso máximo de $\mathrm{O}_{2}$ ya fue descrita en el apartado «Análisis de la dinámica del pulso de $\mathrm{O}_{2}$ ». Como ya se explicó, para esta variable se han formado tres grupos en función de si la dinámica del pulso de $\mathrm{O}_{2}$ era creciente, decreciente o meseta, el pulso máximo de $\mathrm{O}_{2}$ alcanzado por cada grupo se representa en la siguiente gráfica(Ver imagen 16).

En la imagen 16 se ve que el mayor pulso máximo de $\mathrm{O}_{2}$ lo alcanza el grupo decreciente, pero esto no es significativo porque este grupo sólo está formado por un sujeto. La desviación típica fue 3.48 (creciente); 0 (decreciente) y 2.95 (meseta).

No se ha encontrado bibliografía referente a esta relación.

\section{$N^{\circ}$ de días semanales de entrenamiento}

El pulso máximo de $\mathrm{O}_{2}$ tiende a ser mayor cuantos más días entrena el sujeto a la semana, pero esta relación no es muy fuerte. Se han agrupado los sujetos en 5 grupos en función de los días que entrenasen a la semana, el pulso máximo de $\mathrm{O}_{2}$ medio de cada grupo se representa en la gráfica (ver imagen 17 ).

A pesar de que el grupo 1 , tiene el pulso de $\mathrm{O}_{2}$ más alto ( $\mathrm{n}=1$ ), en el resto de los casos se observa que hay una ligera tendencia a aumentar el pulso máximo de $\mathrm{O}_{2}$ con los días de entrenamiento.

Por tanto, podemos creer que aquellos sujetos que entrenan más días a la semana tienen una mayor tendencia a alcanzar un mayor pulso de $\mathrm{O}_{2}$ durante una prueba progresiva máxima, sin embargo este aumento depulso máximo de $\mathrm{O}_{2}$ tampoco es muy grande, por lo que esta variable no es especialmente importante.

No se ha encontrado bibliografía referente a esta relación.

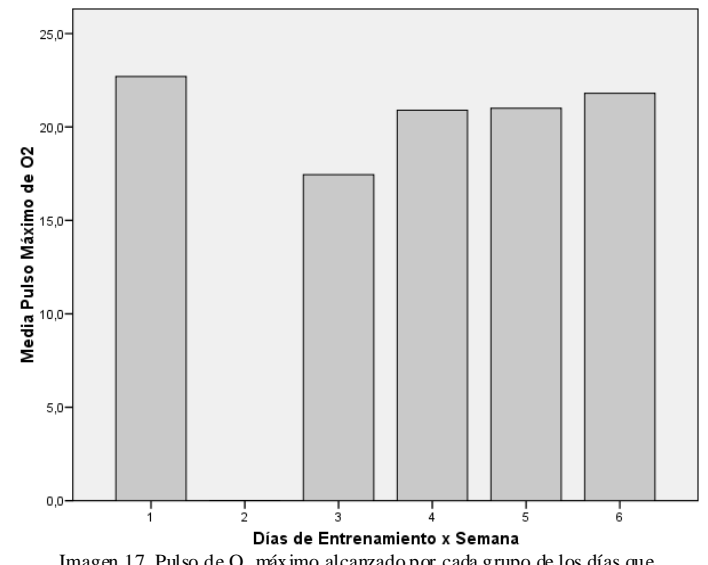
entrenamiento a la semana.

Número de horas de entrenamiento a la semana

Hay una pequeña tendencia a que el pulso máximo de $\mathrm{O}_{2}$ sea más alto en aquellos sujetos que entrenan más días por semana. Para esta relación los sujetos fueron divididos en 6 grupos en función del número total de horas de entrenamiento a la semana (intervalo 2.5), el pulso máximo de $\mathrm{O}_{2}$ medio en cada uno de esos grupos se puede observar en la gráfica (ver imagen 18).

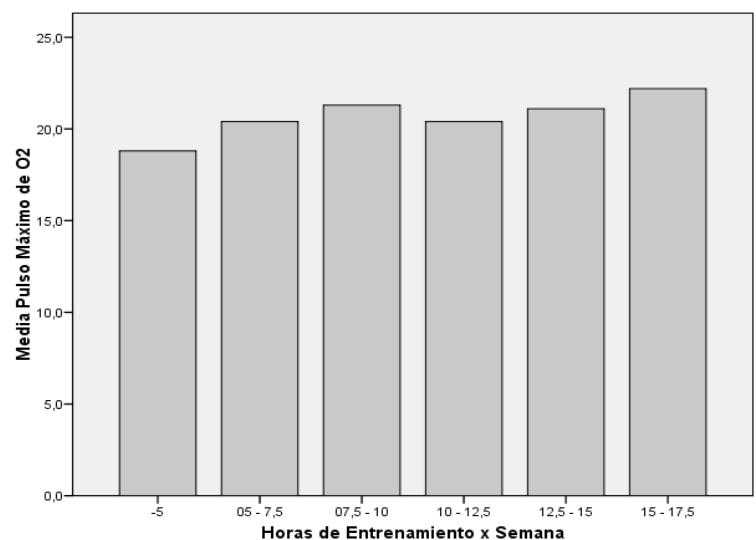

Imagen 18. Pulso máximo de $\mathrm{O}_{2}$ alcanzado por cada grupo en función del número de horas de entrenamiento por semana (intervalo 2.5 ). 
Esta variable podría ser comparada, aunque de una forma poco exacta (ya que puede hacer casos de sujetos que hagan más volumen que otros en menos horas, o que sus entrenamientos tengan una baja intensidad), con el volumen de entrenamiento, el cual sí que parece tener una influencia directa con el pulso máximo de $\mathrm{O}_{2}$ según los estudios ya citados de Padilla et al. (2000), Barbier et al. (2006) y Anastasakis et al. (2005). De todas formas en este caso no se encuentran diferencias tan notables como en estos estudios.

\section{Años que lleva entrenando}

Existe una ligera tendencia a que el pulso máximo de $\mathrm{O}_{2}$ sea más elevado cuantos más años lleve entrenando el sujeto. Los sujetos se han agrupado en 7 grupos en función de los años que llevan entrenando (intervalo3), la media del pulso máximo de $\mathrm{O}_{2}$ alcanzado por cada grupo se expresa en la gráfica (ver imagen 19).

Podemos pensar que lo más lógico sería que aquellos individuos que más años llevan en la competición deberían de tener valores más elevados de pulso máximo de $\mathrm{O}_{2}$, sin embargo, la presencia de los picos nos hace pensar que esta tendencia tampoco es muy importante y, por tanto, se podrían dar casos de individuos que con muy pocos años o incluso meses de entrenamiento, alcancen valores muy elevados de pulso máximo de $\mathrm{O}_{2}$ y viceversa.

No se ha encontrado bibliografía referente a esta relación.

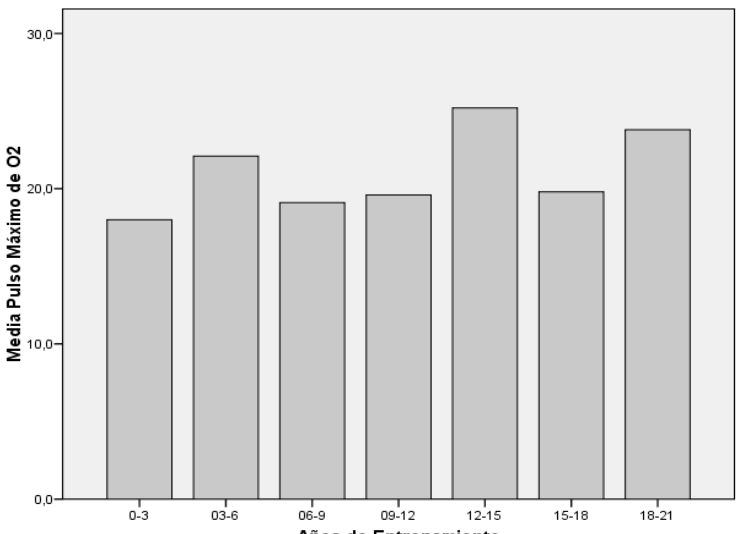

Imagen 19. Pulso máximo de $\mathrm{O}_{2}$ alcanzado por cada grupo en función de los años que llevan los sujetos entrenando (intervalo 3 ).

\section{Conclusiones}

Se concluye que tras esta investigación se han encontrado variables que tienen correlación con el pulso máximo de $\mathrm{O}_{2}$, tales como: sexo, peso, altura e intensidad. Sin embargo, no se ha encontrado correlación con el resto de variables propuestas; modalidad deportiva, edad, volumen de entrenamiento, años en la competición, horas de entrenamiento a la semana, años que se ha estado entrenado y situación competitiva actual.

\section{Referencias}

Anastasakis, A., Kotsiopoulou, C., Rigopuolos, A., Theopistou, A., Protonotarios, N., Panagiotakos, D. \& Stefanadis, C. (2005). Similarities in the profile of cardiopulmonary exercise testing between patients with hypertrophic cardiomyopathy and strength athletes. Scientific Letter, 1477-1478.

Barbier, J., Lebiller, E., Ville, N., Rannou-Bekono, F., \& CarréF. (2006). Relationships between sports-specific characteristics of athlete's heart and maximal oxygen uptake. European Journal of Cardiovascular Prevention \& Rehabilitation, 13(1), 115-121.

Batterham, A., Vanderburgh, P., Mahar, M., \& Jackson, A. (1999). Modeling the influence of body size on $\mathrm{VO}_{2}$ peak: effects of model choice and body composition. Journal of Applied Physiology, 87, 1317-1325.

George, J., Fisher, A. \& Vehrs, P. (2005). Test y pruebas físicas (4 Ed.).Barcelona: Paidotribo.

LeMaitre, J., Harris, S., Hannan, J., Fox, K., \& Denvir, M. (2005). Maximun oxygen uptake corrected for skeletal muscle mass accurately predicts functional improvements following exercise training in chronic heart failure. The European Journal of Heart Failure, 8, 243-248.

Padilla, J., Ojeda, P., Fernández, Y., \& Licea, J. (2000). Pulso máximo de oxígeno en atletas mexicanos de alto rendimiento. Revista del Instituto Nacional de Enfermedades Respiratorias de México, 13(2), 73-84. Disponible en http://www.medigraphic.com/espanol/e-htms/e-iner/ e-in2000/e-in00-2/em-in002b.htm

Pimentel, A., Gentile, C., Tanaka, H., Seals, D., \& Gates, P. (2003). Greater rate of decline in maximal aerobic capacity with age in endurance-trained than in sedentary men. Journal of Applied Physiology, 94, 2406-2413.

Sharma, S., Elliott, P. M., Whyte, G, Mahon, N., Virdee, M. S. \& McKenna, W. J. (2000) Utility of metabolic exercise testing in distinguishing hypertrophic cardiomyopathy from physiologic left ventricular hypertrophy in athletes. Journal of the American College of Cardiology, 36, 864-870.

Woo, J., Derleth, C., Stratton, J., \& Levy, W. (2006). The influence of age, gender and training on exercise efficiency. Journal of the American College of Cardiology, 47(5), 1049-1057.
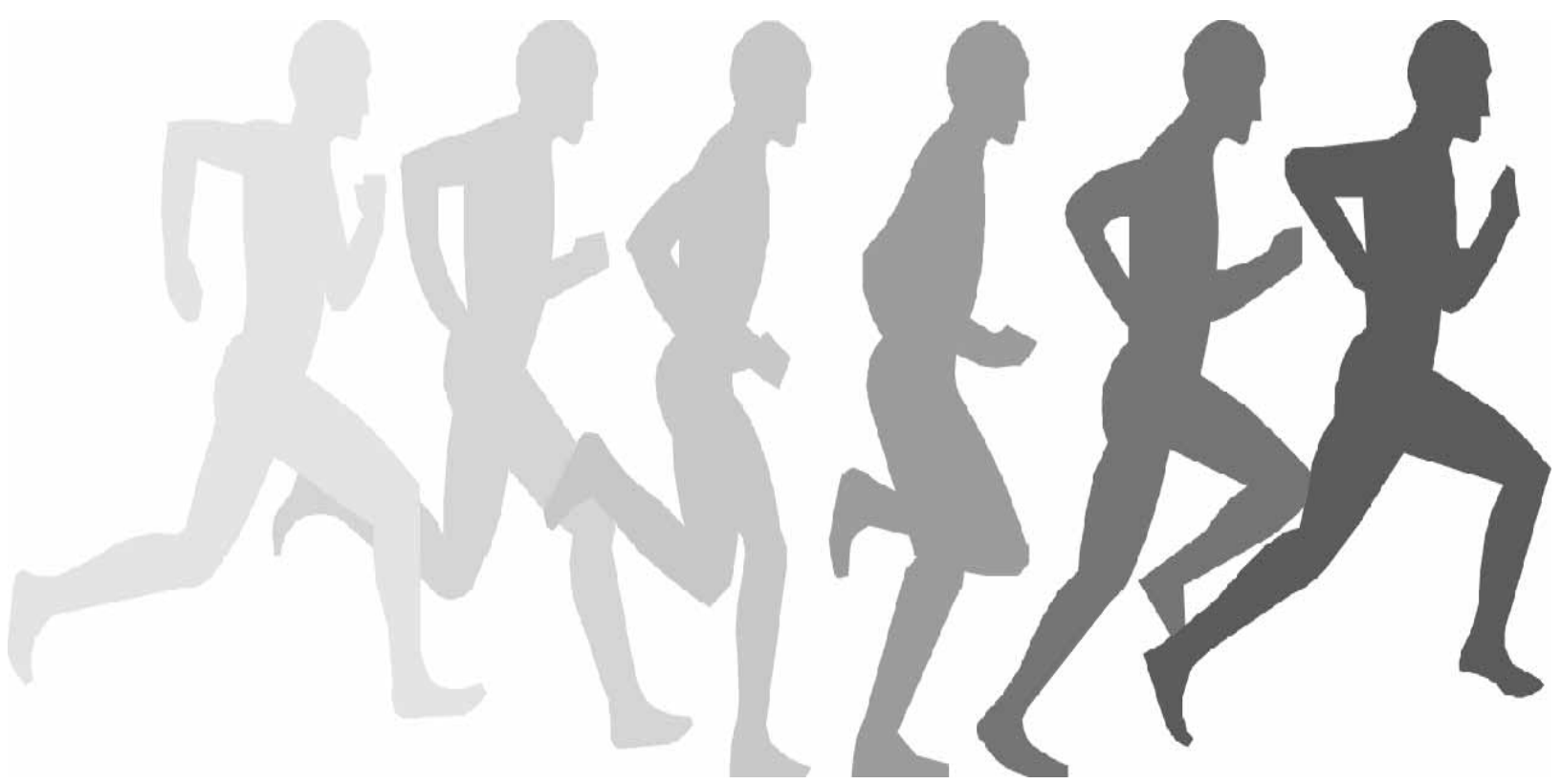